\title{
Surgical outcome for patients with the mitral stenosis- aortic atresia variant of hypoplastic left heart syndrome
}

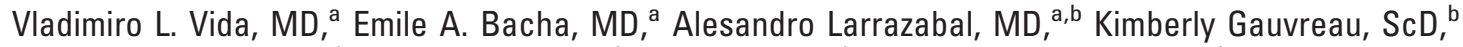 \\ Adam L. Dorfman, MD, ${ }^{\mathrm{b}}$ Gerald Marx, MD, ${ }^{\mathrm{b}}$ Tal Geva, MD, ${ }^{\mathrm{b}}$ Audrey C. Marshall, MD, ${ }^{\mathrm{b}}$ Frank A. Pigula, MD, ${ }^{\mathrm{a}}$ \\ John E. Mayer, MD, ${ }^{a}$ Pedro J. del Nido, MD, and Francis Fynn-Thompson, MDa
}

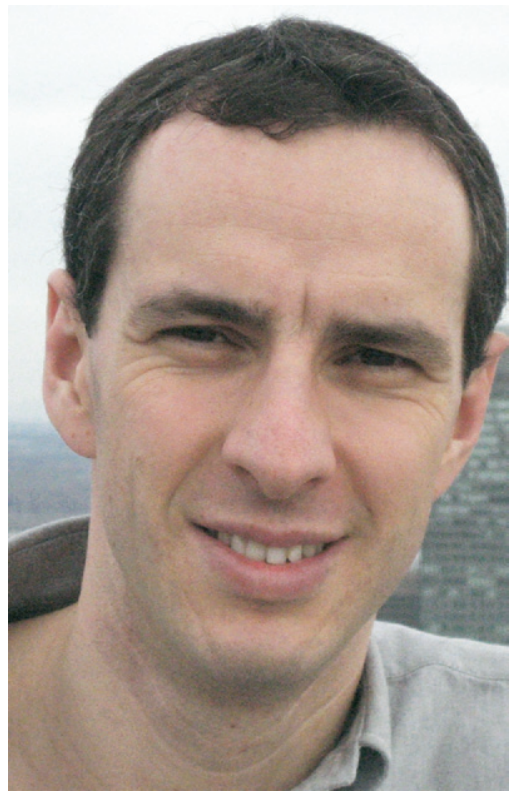

Dr Vida

Objective: We sought to identify and characterize a subgroup of patients with hypoplastic left heart syndrome who might be at higher risk for stage I failure.

Methods: From January 2001 through December 2006, all patients with hypoplastic left heart syndrome who underwent stage I palliation at Children's Hospital Boston were retrospectively reviewed. The subgroup with the mitral stenosis-aortic atresia variant was studied separately. We evaluated preoperative echocardiographic data, operative characteristics, and postoperative factors associated with death or the need for transplantation. The Kaplan-Meier method was used to assess survival.

Results: Thirty-eight (23\%) of 165 patients had mitral stenosis-aortic atresia. Hospital mortality or need for transplantation for patients with mitral stenosis-aortic atresia was significantly higher than for other anatomic subgroups (29\% vs $7.9 \%, P=.002)$. Left ventricle-subepicardial coronary artery communications were present in $20(53 \%)$ patients with mitral stenosis-aortic atresia and were associated with a significantly higher hospital mortality (50\% vs $6 \%, P=.004$ ). No difference in outcome was demonstrated between different sources of pulmonary blood flow. A longer cardiopulmonary bypass time $(P=.02)$ and the need for postoperative extracorporeal membrane oxygenation support $(P<.001)$ were associated with a higher mortality rate.

Conclusions: With improved outcomes in the management of neonates with hypoplastic left heart syndrome, those with the mitral stenosis-aortic atresia variant and left ventricle-subepicardial coronary artery fistulae have emerged as a higher-risk subgroup for failure of stage I palliation. Further investigation is required, and a change in clinical management strategy for this particular subgroup might be warranted.

From the Departments of Cardiac Surgery ${ }^{\mathrm{a}}$ and Cardiology, ${ }^{\mathrm{b}}$ Children's Hospital Boston, Boston, Mass.

Read at the Eighty-seventh Annual Meeting of The American Association for Thoracic Surgery, Washington DC, May 5-9, 2007.

Received for publication May 9, 2007; revisions received Aug 30, 2007; accepted for publication Sept 14, 2007.

Address for reprints: Francis FynnThompson, MD, Department of Cardiac Surgery, Children's Hospital Boston, Harvard Medical School, 300 Longwood Ave, Bader 273, Boston, MA 02115 (E-mail: francis.fynnthompson@cardio.chboston. org).

J Thorac Cardiovasc Surg 2008;135:339-46

0022-5223/\$34.00

Copyright @ $\odot 2008$ by The American Association for Thoracic Surgery

doi:10.1016/j.jtcvs.2007.09.007

$\mathrm{H}$ ospital outcome after stage I surgical palliation for hypoplastic left heart syndrome (HLHS) in our institution and others has improved considerably in the last 2 decades (Figure 1). ${ }^{1,2}$ The decrease in overall hospital mortality for these patients prompted a review of our more recent experience to investigate whether there are specific anatomic subsets of patients who have not benefited from this trend and might still be at increased risk. To date, few studies have looked at the specific anatomic variants of HLHS and their effect on surgical outcomes. ${ }^{1-4}$ Most published studies have focused primarily on other factors, such as weight, prematurity, the size of the ascending aorta, or the relationship of left ventricular (LV) to right ventricular $(\mathrm{RV})$ volumes. ${ }^{1-3}$

In a broader review of contemporary results for surgical palliation of HLHS, we observed worse early outcomes in the subset of patients with mitral stenosis-aortic atresia (MS-AA) when compared with other anatomic variants (Figure 1). This finding had been reported in previous studies but not specifically analyzed. ${ }^{3,5}$ In patients with HLHS and anatomy consisting of MS-AA, previous angiographic and pathologic studies have described the presence of unique coronary artery anomalies, 

Abbreviations and Acronyms
BDG = bidirectional Glenn
$\mathrm{CPB}=$ cardiopulmonary bypass
ECMO = extracorporeal membrane oxygenation
HLHS = hypoplastic left heart syndrome
IAS = intact or highly restrictive atrial septum
$\mathrm{LV}=$ left ventricular
LV-CA = left ventricle-coronary artery
mBTS $=$ modified Blalock-Taussig shunt
MS-AA = mitral stenosis-aortic atresia
$\mathrm{RV}=$ right ventricular
RV-PA = right ventricle-pulmonary artery
SVR = Single Ventricle Reconstruction

identified as left ventricle-subepicardial coronary artery (ventriculocoronary) communications (left ventricle-coronary artery [LV-CA] fistulae). ${ }^{6-8}$ In addition, it has also been demonstrated that in this group of patients, there are more frequent abnormal pathologic findings within the ventricle, such as myocardial necrosis, calcifications, and interstitial fibrosis. ${ }^{9}$

The purpose of this review is to summarize the recent surgical outcomes for patients with HLHS and the MS-AA variant at our institution and to identify factors associated with hospital mortality and failure of stage I Norwood palliation within this subgroup.

\section{Materials and Methods}

Review of medical records and computerized hospital data was approved by the Children's Hospital Committee on Clinical Investigation (institutional review board), and the procedures fol-

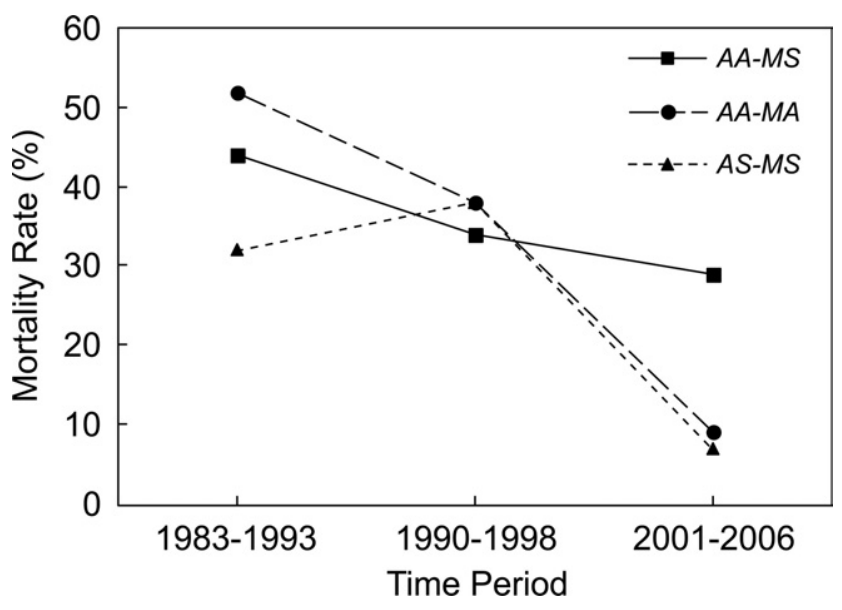

Figure 1. Hospital mortality for stage I palliation in HLHS during the last 2 decades at the Children's Hospital Boston. HLHS, Hypoplastic left heart syndrome; $\boldsymbol{A A}-\boldsymbol{M s}$, aortic atresia-mitral stenosis; $A A-M A$, aortic atresia-mitral atresia; $A S-M S$, aortic stenosis-mitral stenosis. lowed were in accordance with institutional guidelines for retrospective record review and protection of patient confidentiality. The need for patient consent was waived.

As part of a larger review of our contemporary outcomes of stage I palliation for HLHS, we retrospectively identified and included in the study all infants with the diagnosis of HLHS and MS-AA who underwent stage I palliation at the Children's Hospital Boston between January 2001 and December 2006.

\section{Echocardiographic Evaluation}

The anatomic diagnosis of the MS-AA variant of HLHS was identified by means of postnatal echocardiographic analysis in all patients. A further retrospective review of the preoperative echocardiogram was done in all 38 patients with MS-AA. All the echocardiograms were independently reviewed by 2 cardiologists in our institution, focusing on the status of the interatrial septum, measurements of the mitral valve annulus, mitral valve function, LV end-diastolic volume, septal thickness, size of the ascending aorta, and presence of LV-CA fistulae. The echocardiographic presence of LV-CA fistulae was defined as the presence of systolic and diastolic flow across the LV myocardium away from the mitral valve orifice. ${ }^{10-13}$ In the last 3 patients in our series, we confirmed the echocardiographic diagnosis of LV-CA fistulae with angiographic analysis. The echocardiographic findings in these 3 catheterized patients with angiographically documented fistulae were used during the retrospective review of echocardiograms in the remaining 35 patients with MS-AA (Figure 2).

The currently accepted definition of intact or highly restrictive atrial septum (IAS) was used as inclusion criteria for the definition of this group of patients within our cohort of study ${ }^{14-16}$ and included patients for whom the following was the case: (1) the atrial septum is intact on either a prenatal or postnatal echocardiogram, (2) a prenatal echocardiogram showed a small atrial septal defect with prominent pulmonary venous flow reversal, or (3) the neonate had to be taken on an urgent or emergency basis to the catheterization laboratory for left atrial decompression because of clinical signs of left atrial hypertension (severe hypoxia, hemodynamic instability, or both).

\section{Surgical Procedure}

All patients were taken to the operating room after resuscitation and inotropic and ventilatory support, as necessary. Surgical intervention consisted of standard stage I Norwood palliation with neoaortic arch reconstruction and atrial septectomy. Pulmonary blood flow was restored with either a modified Blalock-Taussig shunt (mBTS) or a right ventricle-pulmonary artery (RV-PA) conduit based on the individual surgeon's preference and on randomization in the ongoing Single Ventricle Reconstruction (SVR) trial since January 2004. A period of deep hypothermic circulatory arrest, selective antegrade cerebral perfusion, or both was used in all cases at the surgeon's discretion.

\section{Statistical Analysis}

The primary outcome variables were failure of stage I palliation (defined as in-hospital mortality or need for heart transplantation) and interstage mortality occurring between stage I palliation and bidirectional Glenn (BDG) shunt. Patient and procedural variables assessed for association with these outcomes included prenatal 
TABLE 1. Variables associated with failed stage I palliation (mortality/transplantation)

\begin{tabular}{|c|c|c|c|}
\hline & Survivors $(\mathrm{n}=27)$ & Failed stage I $(\mathrm{n}=11)^{*}$ & $P$ value \\
\hline \multicolumn{4}{|l|}{ Significant variables } \\
\hline LV-CA fistulae, n (\%) & $10(37)$ & $10(91)$ & .004 \\
\hline Mean CPB time, $\mathrm{n}$ (SD) & $138 \pm 28$ & $212 \pm 83$ & .01 \\
\hline Postoperative ECMO, n (\%) & $1(4)$ & $8(73)$ & $<.001$ \\
\hline \multicolumn{4}{|l|}{ Nonsignificant variables } \\
\hline Prenatal diagnosis, n (\%) & $14(52)$ & $7(64)$ & .72 \\
\hline Prematurity, n (\%) & $2(7)$ & $1(9)$ & 1.0 \\
\hline Chromosomal anomalies, n (\%) & $1(4)$ & $0(0)$ & 1.0 \\
\hline Intact/highly restrictive AS, $\mathrm{n}(\%)$ & $4(15)$ & $5(45)$ & .12 \\
\hline Median age at surgical intervention, $\mathrm{d}$ (range) & $4(0-36)$ & $4(1-8)$ & .79 \\
\hline Median weight, kg (range) & $3.1(2-4.4)$ & $3.1(1.7-4.3)$ & 1.0 \\
\hline mBTS, n (\%) & $15(56)$ & $8(73)$ & 0.47 \\
\hline RV-PA conduit, n (\%) & $12(44)$ & $3(27)$ & .47 \\
\hline Mean CA time, n (SD) & $35 \pm 19$ & $50 \pm 33$ & .18 \\
\hline Shunt revision, n (\%) & $2(7)$ & $3(27)$ & .13 \\
\hline
\end{tabular}

$\angle V$-CA, Left ventricle-coronary artery; $C P B$, cardiopulmonary bypass; ECMO, extracorporeal membrane oxygenation; $A S$, atrial septum; $m B T S$, modified Blalock-Taussig shunt; $R V-P A$, right ventricle-pulmonary artery; $C A$, circulatory arrest. *Including patients with failed stage I palliation who required heart transplantation.

diagnosis, presence of intact or highly restrictive atrial septal communication, anatomic subgroup, presence of coronary artery fistulae, degree of tricuspid valve regurgitation, age at surgical intervention, weight at surgical intervention, type of pulmonary blood flow supply, need for revision of pulmonary blood flow supply, cardiopulmonary bypass (CPB) time, circulatory arrest time, and postoperative extracorporeal membrane oxygenation (ECMO) requirement (Table 1). Continuous variables were summarized as means \pm standard deviation or median (range). Characteristics were compared for patients who died in the hospital after the stage I procedure versus those who survived until discharge by using the Fisher exact test for categorical variables and either the 2-sample $t$ test or the Wilcoxon rank sum test for continuous variables. Patients with and without LV-CA fistulae were compared in a similar manner. Survival probabilities were estimated by using the Kaplan-Meier method.

\section{Results}

\section{Demographics and Baseline Characteristics}

From January 2001 through December 2006, 38 neonates (25 male and 13 female neonates) with HLHS and the MS-AA anatomic subtype presented to our center and underwent stage I palliation. There were no patients with this diagnosis who died while awaiting surgical intervention during this study.

This group with MS-AA represents 23\% (38/165) of all neonates with HLHS presenting to our institution during the same period. Of these 38 patients comprising the study group, $21(55 \%)$ were given prenatal diagnoses of HLHS. Twenty (53\%) of the 38 patients had preoperative echocardiographic evidence of LV-CA fistulae. Of these 38 patients, $9(26 \%)$ had an IAS and underwent creation or enlargement of an atrial septal defect in the catheterization laboratory before surgical intervention.

\section{Stage I Palliation}

The 38 patients comprising the study group had stage I palliation at a median of 4 days (range, $0-36$ days) of age, with a median weight of 3.1 kilograms (range, $1.7-4.4 \mathrm{~kg}$ ). An mBTS supplied pulmonary blood flow in $23(61 \%)$ patients, and its diameter was $3.5 \mathrm{~mm}$ in 20 patients, $4 \mathrm{~mm}$ in 1 patient (with IAS), and $3 \mathrm{~mm}$ in 2 patients who weighed less than $2 \mathrm{~kg}$. When an RV-PA conduit was placed, the conduit was constructed of either a $4-m m(n=1)$ or $5-m m(n=14)$ Gore-Tex tube. The infant who received a 4-mm RV-PA conduit weighed less than $2 \mathrm{~kg}$. In 15 (40\%) patients the source of pulmonary blood flow had been randomized in the ongoing SVR trial (mBTS, 5; RV-PA conduit, 10).

Mean CPB time was $160 \pm 59$ minutes, mean crossclamp time was $43 \pm 32$ minutes, and mean circulatory arrest time was $40 \pm 24$ minutes. The chest was initially left open in $33(87 \%)$ patients after surgical palliation and was closed after a median of 3 days (range, 2-5 days).

Four (11\%) of 38 patients required shunt revision ( 3 of these patients had an mBTS). Excessive pulmonary blood flow prompted anatomic reduction of the shunt diameter in 2 patients, whereas acute shunt thrombosis occurred in 1 patient. In the remaining patient the RV-PA conduit was changed to an mBTS because of the finding of stenosis at the proximal conduit anastomosis associated with a severely thickened right ventricle.

Nine (24\%) patients were supported postoperatively with ECMO; 6 of them died, and 2 underwent transplantation. Indications for ECMO included inability to wean from $\mathrm{CPB}$ $(\mathrm{n}=3)$, severe RV dysfunction $(\mathrm{n}=3)$, cyanosis $(\mathrm{n}=2)$, and acute shunt thrombosis $(\mathrm{n}=1)$. 
TABLE 2. Variables associated with the presence of fistulae

\begin{tabular}{|c|c|c|c|}
\hline & \multicolumn{2}{|c|}{ Fistulae } & \multirow[b]{2}{*}{$P$ value } \\
\hline & Yes $(n=20,53 \%)$ & No $(n=18,47 \%)$ & \\
\hline \multicolumn{4}{|l|}{ Significant variables } \\
\hline Failed stage I palliation, n $(\%)^{*}$ & $10(50)^{*}$ & $1(6)$ & .004 \\
\hline Mean CPB time, n (SD) & $181 \pm 71$ & $135 \pm 29$ & .01 \\
\hline \multicolumn{4}{|l|}{ Nonsignificant variables } \\
\hline Prenatal diagnosis, $\mathrm{n}(\%)$ & $11(55)$ & $10(56)$ & 1.0 \\
\hline Prematurity, $\mathrm{n}(\%)$ & $3(15)$ & $0(0)$ & .23 \\
\hline Chromosomal anomalies, n (\%) & $1(4)$ & $0(0)$ & 1.0 \\
\hline Intact/highly restrictive AS, n (\%) & $8(40)$ & $2(11)$ & .07 \\
\hline Median age at surgical intervention, $d$ (range) & $4.5(1-36)$ & $4(0-15)$ & .69 \\
\hline Median weight, $\mathrm{kg}$ (range) & $3.1(1.7-4.3)$ & $3(2-4.4)$ & .91 \\
\hline mBTS, n (\%) & $8(40)$ & $7(39)$ & 1.0 \\
\hline RV-PA conduit, n (\%) & $12(60)$ & $11(61)$ & 1.0 \\
\hline Mean CA time, $\mathrm{n}$ (SD) & $42 \pm 28$ & $37 \pm 19$ & .48 \\
\hline Shunt revision, n (\%) & $3(15)$ & $2(11)$ & 1.0 \\
\hline Postoperative ECMO, n (\%) & $7(35)$ & $2(11)$ & .13 \\
\hline
\end{tabular}

$C P B$, Cardiopulmonary bypass; $S D$, standard deviation; $A S$, atrial septum; $m B T S$, modified Blalock-Taussig shunt; $R V$ - $P A$, right ventricle-pulmonary artery; $C A$, circulatory arrest; $E C M O$, extracorporeal membrane oxygenation. *Including patients with failed stage I palliation who required heart transplantation.

\section{Hospital Survival and Predictors of Mortality}

Hospital mortality and need for transplantation for patients with MS-AA was significantly higher than those for the other anatomic subgroups of patients with HLHS undergoing stage I palliation during the same period (29\% vs $7.9 \%$, $P=.002$ ). Twenty-seven patients survived stage I palliation. Seven patients died from RV failure, 3 from sepsis, and 1 from alveolar capillary dysplasia. Two additional patients underwent orthotopic heart transplantation after stage I palliation failed; 1 survived, and 1 did not. For the 27 hospital survivors to discharge, median hospital stay was 20 days (range, 4-82 days). The presence of LV-CA fistulae was associated with failure of stage I palliation $(P=.004)$. Other variables significantly associated with hospital mortality were longer $\mathrm{CPB}$ time $(P=.02)$ and postoperative ECMO requirement $(P<.001$, Tables 1 and 2$)$. Four patients, all with LV-CA fistulae, required prolonged $\mathrm{CPB}$ assistance in the operating room because of multiple failed attempts of weaning; in 3 patients ECMO support was started in the operating room. The presence of IAS or highly restrictive atrial septum was not significantly associated with increased hospital mortality $(P=.12)$ in patients with MS-AA.

\section{Interstage and Midterm Survival}

Twenty-four (83\%) of the 27 stage I survivors underwent a BDG procedure at a median of 138 days after stage I palliation (range, 58-298 days). Interstage mortality occurred in $1(3 \%)$ patient. The cause of mortality was RV dysfunction (this patient had an initial mBTS). This resulted in an overall pre-BDG (hospital and interstage) mortality rate of $31.5 \%$ (12/38 patients).

There was no hospital mortality associated with BDG procedures. One patient died 262 days after a BDG proce-
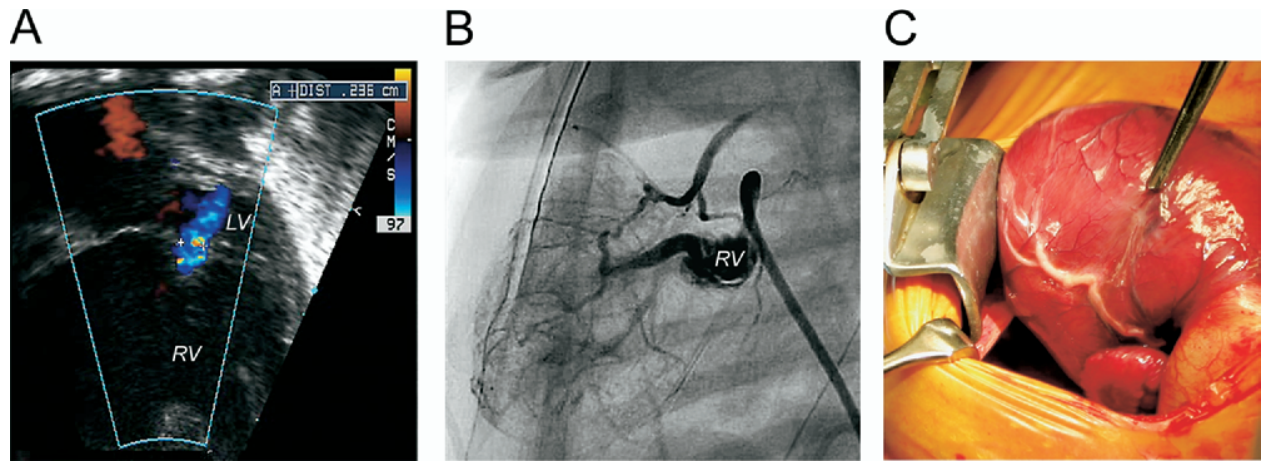

Figure 2. Echocardiographic (A), angiographic (B), and intraoperative (C) images of left ventricle-coronary artery fistulae. $L V$, Left ventricle; $R V$, right ventricle. 


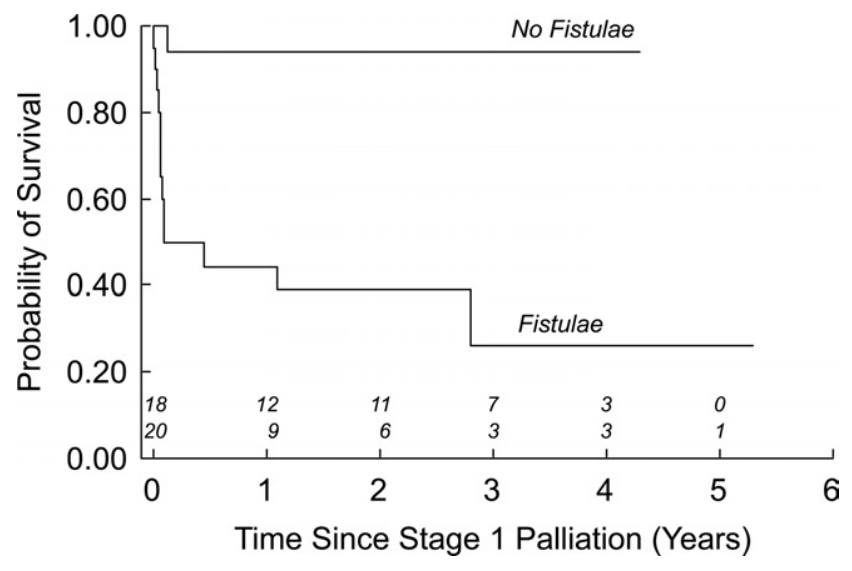

Figure 3. Kaplan-Maier survival estimates according to the presence of left ventricle-coronary artery fistulae (log-rank test for equality of survival functions: $P<.001$ ).

dure of RV failure. Two patients are still waiting for BDG procedures. At most recent follow-up, 14 patients had undergone a fenestrated lateral tunnel Fontan operation at a median age of 31.3 months (range, 20.4-44.1 months). Survival estimates according to the presence of LV-CA fistulae are shown in Figure 3.

\section{Discussion}

As with other large-volume centers, the hospital survival after stage I Norwood palliation for HLHS has dramatically increased in our institution over the last 2 decades (Figure 1). This improvement in survival can be attributed to a number of factors, including advances in perioperative and operative strategies, as well as progress in transcatheter techniques.

We undertook the current review in an attempt to determine specific survival rates and predictors of mortality in recent years. The overall hospital survival at the Children's Hospital Boston after stage I palliation during the last 6 years is $89 \%$ and is comparable with the recent published data in the literature. ${ }^{4,5,17,18}$ However, the risk of death and failure of stage I palliation is not homogenously distributed across the entire cohort of patients with HLHS, and patients with the MS-AA subtype and LV-CA fistulae had the highest risk of hospital mortality and failure of stage I palliation $(P=.002)$.

In 1990, Murdison and colleagus ${ }^{19}$ reported that the MS-AA subgroup faired worse in the short and intermediate term after surgical palliation than other anatomic subgroups of HLHS. Subsequently, Jonas and associates, ${ }^{3}$ in 1994, speculated about a possible correlation between the diagnosis of MS-AA and increased hospital mortality after stage I palliation. Sugiyama and coworkers ${ }^{9}$ found that patients with MS-AA had a thicker endocardium, and, histologi- cally, there were more abnormal findings, such as myocardial necrosis, calcification, and interstitial fibrosis, compared with other subtypes. These authors speculated that these findings were the result of relative coronary hypoperfusion at the time of surgical intervention. Sauer and colleagues, ${ }^{8}$ in 1989, also reported a pathologic study demonstrating the association of coronary artery anomalies in $42 \%$ of the hearts of patients with HLHS and MS-AA. They observed that the hearts of these patients had a higher percentage of endocardial fibroelastosis, as well as thicker and more tortuous coronary arteries with an increased intimal thickness and stenosis, especially in the proximal left coronary artery. This finding was suggestive of the presence of anatomically significant fistulae. They theorized that these coronary abnormalities might impair ventricular function.

Our study demonstrated that patients with MS-AA had a significantly higher mortality when compared with that of other patients with HLHS undergoing stage I palliation. However, on closer analysis, this increased risk appears to be limited to patients with associated LV-CA fistulae. None of the other studied variables were associated with significantly worse outcomes. In particular, the presence of IAS or highly restrictive atrial septum, although prevalent in this subgroup with MS-AA, was not related to hospital outcome in our analysis. This might be due to the small number of patients included in the study and will have to await confirmation by other studies.

The exact mechanisms for the increased rate of failure after stage I palliation in patients with MS-AA and fistulae are still unclear. We hypothesize that the presence of fistulae might interfere with adequate myocardial protection during CPB or might result in coronary hypoperfusion once the heart is decompressed on CPB. The fact that many of the patients who died had problems beginning in the operating room related to CPB (resulting in significantly longer bypass times for the group that died) possibly suggests such a mechanism. However, those hypotheses remain unproved.

In conclusion, the presence of LV-CA fistulae in patients with HLHS and MS-AA is a risk factor for increased hospital mortality after surgical palliation. Patients with this anatomic variant of HLHS represent an identifiable subgroup that is at significantly increased risk. Based on these findings, we have changed the institutional policy for these patients, performing preoperative angiography on all patients with MS-AA to confirm and better delineate the LV-CA fistulae. Further prospective evaluations with larger numbers of patients are required to recommend modification of the current surgical management of this anatomic variant of HLHS. Possible surgical alternatives include neonatal hybrid stage I palliation and heart transplantation. 


\section{References}

1. Daebritz SH, Nollert GD, Zurakowski D, Khalil PN, Lang P, del Nido PJ, et al. Results of Norwood stage I operation: comparison of hypoplastic left heart syndrome with other malformations. J Thorac Cardiovasc Surg. 2000;119:358-67.

2. Forbess JM, Cook N, Roth SJ, Serraf A, Mayer JE Jr, Jonas RA Ten-year institutional experience with palliative surgery for hypoplastic left heart syndrome. Risk factors related to stage I mortality. Circulation. 1995;92:II262-6.

3. Jonas RA, Hansen DD, Cook N, Wessel D. Anatomic subtype and survival after reconstructive operation for hypoplastic left heart syndrome. J Thorac Cardiovasc Surg. 1994;107:1121-8.

4. Stasik CN, Gelehrter S, Goldberg CS, Bove EL, Devaney EJ, Ohye RG. Current outcomes and risk factors for the Norwood procedure. J Thorac Cardiovasc Surg. 2006;131:412-7.

5. Weinstein S, Gaynor JW, Bridges ND, Wernovsky G, Montenegro LM, Godinez RI, et al. Early survival of infants weighing 2.5 kilograms or less undergoing first-stage reconstruction for hypoplastic left heart syndrome. Circulation. 1999;100:II167-70.

6. Baffa JM, Chen SL, Guttenberg ME, Norwood WI, Weinberg PM Coronary artery abnormalities and right ventricular histology in hypoplastic left heart syndrome. J Am Coll Cardiol. 1992;20:350-8.

7. O'Connor WN, Cash JB, Cottrill CM, Johnson GL, Noonan JA Ventriculocoronary connections in hypoplastic left hearts: an autopsy microscopic study. Circulation. 1982;66:1078-86.

8. Sauer U, Gittenberger-de Groot AC, Geishauser M, Babic R, Buhlmeyer K. Coronary arteries in the hypoplastic left heart syndrome. Histopathologic and histometrical studies and implications for surgery. Circulation. 1989;80:I168-76.

9. Sugiyama H, Yutani C, Iida K, Arakaki Y, Yamada O, Kamiya T. The relation between right ventricular function and left ventricular morphology in hypoplastic left heart syndrome: angiographic and pathological studies. Pediatr Cardiol. 1999;20:422-7.

10. Bensky AS, Covitz W. Echocardiographic demonstration of a ventriculocoronary artery communication in a neonate with hypoplastic left heart syndrome. J Am Soc Echocardiogr. 1994;7:324-6.

11. Chaoui R, Tennstedt C, Goldner B. Prenatal diagnosis of ventriculocoronary arterial fistula in a fetus with hypoplastic left heart syndrome and aortic atresia. Ultrasound Obstet Gynecol. 2002;20:75-8.

12. Patel CR, Lane JR, Spector ML, Smith PC, Waight DJ. Prenatal diagnosis of ventriculocoronary arterial communication in fetuses with hypoplastic left heart syndrome. J Ultrasound Med. 2006;25:245-9.

13. Velvis H, Schmidt KG, Silverman NH, Turley K. Diagnosis of coronary artery fistula by two-dimensional echocardiography, pulsed Doppler ultrasound and color flow imaging. J Am Coll Cardiol. 1989;14:968-76.

14. Better DJ, Apfel HD, Zidere V, Allan LD. Pattern of pulmonary venous blood flow in the hypoplastic left heart syndrome in the fetus Heart. 1999;81:646-9.

15. Taketazu M, Barrea C, Smallhorn JF, Wilson GJ, Hornberger LK. Intrauterine pulmonary venous flow and restrictive foramen ovale in fetal hypoplastic left heart syndrome. J Am Coll Cardiol. 2004;43: $1902-7$.

16. Vida VL, Bacha EA, Thiagaragan R, Gauvreau K, Larrazabal LA, Fynn-Thompson F, et al. Hypoplastic left heart syndrome with intact or highly restrictive atrial septum: surgical experience from a single center. Ann Thorac Surg. 2007;84:581-6.

17. Gaynor JW, Mahle WT, Cohen MI, Ittenbach RF, DeCampli WM, Steven JM, et al. Risk factors for mortality after the Norwood procedure. Eur J Cardiothorac Surg. 2002;22:82-9.

18. Mahle WT, Spray TL, Wernovsky G, Gaynor JW, Clark BJ 3rd. Survival after reconstructive surgery for hypoplastic left heart syndrome: a 15-year experience from a single institution. Circulation. 2000;102:III136-41.

19. Murdison KA, Baffa JM, Farrell PE, Chang AC, Barber G, Norwood WI, et al. Hypoplastic left heart syndrome: outcome after initial reconstruction and before modified Fontan procedure. Circulation. 1990;82(suppl IV):IV199-207.

\section{Discussion}

Dr Thomas L. Spray (Philadelphia, Pa). Although great improvements in results for stage 1 Norwood procedures have been achieved over the past decade, there continues to be a very significant mortality with this procedure, and the cause of this early mortality remains frustratingly obscure. Many centers have noted higher mortality associated with certain anatomic and genetic risk factors, such as aortic atresia, associated cardiac or noncardiac anomalies, low birth weight, and so on, but this compelling study adds a new and important risk group: patients with aortic atresia, mitral stenosis, and associated coronary fistulae from a small left ventricle. Although these patients represent a minority of the total group of patients with HLHS, they appear to be a major and, perhaps in your series, the major source of mortality, at least in Boston.

Stimulated somewhat by this article, I went back and looked at an experience from our own center over about a 3-year period ending in May 2005 when we began the SVR trial randomization. Of 176 stage 1 operations in that 3 -year period, our incidence of aortic atresia/mitral stenosis was about $12 \%$, and therefore a little bit less than what you saw in Boston in your own experience. When I got the comparison mortality data for all of those patients, the overall survival was $92 \%$ in the patients without aortic atresia/ mitral stenosis and $84 \%$ in those with aortic atresia/mitral stenosis. Although the numbers are very small, there were only 19 patients with aortic atresia/mitral stenosis, and that is probably not a statistically significant difference, or at least it leaves some credence to the finding that you have noted here.

Interestingly, though, at about a year, the overall survival rates in the 2 groups were the same, and there was no interstage mortality in the group with aortic atresia and mitral stenosis, which again is somewhat surprising.

I have several questions for the authors. First, are coronary fistulae limited to this subgroup, to the patients with aortic atresia/ mitral stenosis? Do they occur if there is a small ventricular septal defect or some other decompressing mode of the left ventricle? Apparently, they still do occur if you have mitral regurgitation, as you noted, which you would think would be a decompressing force on the left ventricle.

How accurate is echocardiography in making this diagnosis? Are you looking for this in every patient? Do you ever see it in the other subgroups? How specific is this?

I must say that in our own center, our echocardiography folks occasionally mention this, but they do not seem to go to great lengths to try to look for these fistulae. I wonder, is that different in Boston?

Dr Vida. Thank you very much for all your questions.

With regard to your first question, we did not study the presence of fistulae at the preoperative echocardiographic analysis in other subgroups of patient with HLHS. We reviewed a number of pathologic reports in the literature that demonstrated that the presence of coronary fistula or left ventricle-coronary artery communication was basically limited to patients with the aortic atresia/ mitral stenosis variant of HLHS.

In response to your second question, we have considered the presence of a ventricular septal defect as a possible differential diagnosis in this group of patients because the fistulae are most often localized in the region of the ventricular septum. However, 
as reported in the literature, the exact nature of the flow pattern in the fistulae is a critical element of diagnostic accuracy. The flow in the coronary fistulae was found to be from the ventricle to the coronary artery in systole and from the coronary artery to the ventricle in diastole, and therefore there was to-and-fro flow across the fistula. In the case of a ventricular septal defect, you can have only have flow going from the left ventricle to the right ventricle, both in systole and diastole.

With regard to the sensitivity of echocardiography as a diagnosis tool, we were quite surprised to find that there was $92 \%$ (35/38 patients) consensus in the diagnosis of fistulae between the 2 separate senior echocardiographers who blindly reviewed the preoperative echocardiographic studies of all the patients in our study. Interestingly, one can make a comparison to the diagnosis of fistulae in patients with pulmonary atresia and intact ventricular septum, where echocardiography is $90 \%$ accurate in patients who are later confirmed to have coronary fistulae by means of angiographic analysis. In conclusion, we think that even though we cannot analyze the number, the magnitude, and the significance of these fistulae, we can diagnose their presence, and this was found to be significantly related to a worse hospital outcome.

Dr Spray. You sort of lead into my second question with that reference. Most of the mortality was early, early after surgical intervention in your series, and most of the deaths seemed to be from complications, to some extent, of RV dysfunction. I mean, even the sepsis is sort of related to a prolonged hospitalization, suggesting that intraoperative events are causative of this poor outcome.

Because the majority of these patients can be expected to have a right coronary-dominant coronary pattern based on previous anatomic studies of this subtype of patients and because in your own institution patients with pulmonary atresia and intact ventricular septum and right ventricle-dependent coronary circulation do not seem to have such early ventricular dysfunction or dysfunction at the time of shunt procedures or Fontan operations, do you think there are potentially other anatomic features that might not have been assessed here? The smaller aorta might not be effective, but what about tricuspid regurgitation? You did not mention that as one of the variables. What about preoperative RV dysfunction; was that present? That might account for some of these differences in outcome.

Dr Vida. As discussed, we analyzed various anatomic variables in these patients without finding any significant difference between those patients with and without coronary fistulae. What we found is that 7 of the 11 patients who died or had a failed stage 1 procedure had RV failure, and the possible mechanism of death in these patients was myocardial ischemia. Five of the patients had problems being weaned off $\mathrm{CPB}$, and 3 patients required ECMO support in the operating room.

We are now prospectively studying all patients with a diagnosis of HLHS and aortic atresia/mitral stenosis who had an echocardiographic diagnosis of coronary fistulae with angiography to understand whether there are other anatomic variables that we are missing.

We have 5 patients who have a preoperative angiographic confirmation of coronary fistulae: 3 were included in this study, and 2 were not included because they did not have a traditional stage I palliation but rather underwent a hybrid stage 1 procedure. Both are alive and were discharged home.

We also reviewed the routine cardiac angiography that is performed before the BDG procedure. Only 8 patients had a left ventriculography, and in 2 of the 3 patients with the preoperative diagnosis of coronary fistulae, there was complete regression of these fistulae. Therefore this again is something that needs to be considered for further study.

Dr Spray. Did you do any posts on the patients who died? If you did, did you find any common mechanism or common pattern of injury? Were these ventricular dysfunctions because of ischemic damage?

Dr Vida. There were 4 patients who had an autopsy; 2 of them are heart specimens obtained at the time of the transplantation. The only remarkable thing that we have found was the presence of atresia of the left coronary ostium with aortocoronary discontinuation in 1 patient. All the blood flow to the left coronary artery was provided by the huge fistula between the left ventricle and the left coronary artery. In all the other patients, we found signs of ischemia with infarcted myocardium.

Dr Spray. I think this study should stimulate other centers to go back and analyze their own patient groups. I think it is hopeful that data from the ongoing SVR trial, which is a randomized trial with a much larger multi-institutional patient population, will be able to separate out these characteristics and support these interesting findings.

Dr Christian Pizarro (Wilmington, Del). That was a great presentation.

What was the source of pulmonary blood flow in these patients? Were they evenly distributed between Blalock-Taussig shunts and RV-PA conduct?

Dr Vida. The source of pulmonary blood flow was an mBTS in 23 patients and an RV-PA conduit in 15 patients. Before 2004, the source of pulmonary blood flow was chosen according to surgeon's preference, whereas since January 2004, it has been randomized according to the SVR trial.

Dr Pizarro. Did you observe any difference?

Dr Vida. No, there was no difference in hospital outcome according to the type of pulmonary blood flow in this group of patients.

Dr Pizarro. Not at all?

Dr Vida. No.

Dr Pizarro. How do you explain the difference in the length of bypass between the patients who did well and the ones who did not? Did you change the shunt, or did you do anything about it?

Dr Vida. As previously discussed, 5 of the 11 patients who had a bad outcome needed to go back on bypass because of hemodynamic instability and signs of myocardial ischemia characterized by ST-segment changes at echocardiography. In 1 patient we had to redo the Damus-Kaye-Stansel anastomosis because we thought the problem was residual arch obstruction with possible coronary involvement, but that was not the case. Therefore we believe that the prolonged CPB times in patients who died or had a failed stage I procedure were due to myocardial ischemia after the surgical palliation caused by the coronary fistulae.

Dr Pizarro. I would just caution you regarding your last conclusion. In reference to the hybrid procedure, I think there is at least anecdotal experience to suggest that the physiology of a 
hybrid procedure is very similar to that in patients who undergo a stage 1 Norwood procedure with a Blalock-Taussig shunt, and therefore the problematic issue regarding coronary perfusion remains. Although it is a lesser intervention, I think the physiology is probably not as favorable.

Dr Vida. We believe that the main advantage of the hybrid procedure is the avoidance of the CPB and crossclamp times. The avoidance of myocardial ischemia might be a key point in explaining the better outcome in certain patients in our series.

Dr Christian Brizard (Melbourne, Australia). I have 2 short questions.

I wonder whether you have demonstrated a difference of size in the left ventricle and whether that would give a hint toward a left and right ventricle interaction in this group of patients.

Second, I understand that you have now a strategy to demonstrate preoperatively coronary artery fistulae. If you do demonstrate coronary fistulae, what do you do differently?

Dr Vida. With regard to your first question, one of the first variables we have analyzed in exploring the higher mortality in this group of patients was the size of the left ventricle and its interaction with the motion of the ventricular septum. However, we did not find any difference in size between patients with fistulae and those without fistulae. Again, there was no difference in the size of the left ventricle between those patients who died and those who survived the stage I palliation. In addition, we tried to correlate the $\mathrm{Z}$-score of the mitral valve with the presence of fistulae, like comparing the Z-score of the tricuspid valve and the presence of fistulae in patients with pulmonary atresia and intact ventricular septum, but again, no relationship has been found.

In response to your second question, we have a new policy in our institution to perform cardiac angiography, with LV injection in all patients with preoperative echocardiographic diagnosis of coronary fistulae. If the presence of fistulae is confirmed by means of angiographic analysis, we now consider alternative management strategies, such as the hybrid procedure and heart transplantation. We have performed the hybrid procedure in 2 patients with these characteristics, and both were discharged home and are alive. The role of primary heart transplantation in these patients is still a much debated point.

Dr Emile A. Bacha (Boston, Mass). I have just a quick addendum to Dr Spray's comment about what he found with the Philadelphia patients. It is very important to point out that we believe that it is the fistulae per se that are the problem. If you do not look for the LV coronary fistulae, you might not really look at the root of the problem. Aortic atresia/mitral stenosis alone in our series without fistulae was not tied with a higher mortality at all. Therefore if you have aortic atresia/mitral stenosis per se without fistulae, your survival is about the same, but if you have aortic atresia/mitral stenosis with fistulae - and I would stress the point that you have to look for them-survival is worse. It is really something that one has to look for, either by means of echocardiographic analysis or by means of catheterization. Once we started looking for them, it emerged as one of the risk factors, buttressed by some of the pathology data that Vlad mentioned. That is a very important point not to be confused with just aortic atresia/mitral stenosis alone. 